\title{
The study of synchronization of rhythms of microvascular blood flow and oxygen saturation during adaptive changes
}

\author{
Andrey V. Dunaev, Victor V. Sidorov, Alexander I. \\ Krupatkin M.D., Ilya E. Rafailov, Scott G. Palmer, et al.
}

Andrey V. Dunaev, Victor V. Sidorov, Alexander I. Krupatkin M.D., llya E. Rafailov, Scott G. Palmer, Sergei G. Sokolovski, Neil A. Stewart, Edik U. Rafailov, "The study of synchronization of rhythms of microvascular blood flow and oxygen saturation during adaptive changes," Proc. SPIE 8935, Advanced Biomedical and Clinical Diagnostic Systems XII, 89350A (27 February 2014); doi: $10.1117 / 12.2035748$ 


\title{
The study of synchronization of rhythms of microvascular blood flow and oxygen saturation during adaptive changes
}

\author{
Andrey V. Dunaev*a, ${ }^{\text {a,b }}$, Victor V. Sidorov ${ }^{\mathrm{c}}$, Alexander I. Krupatkin ${ }^{\mathrm{d}}$, Ilya E. Rafailov ${ }^{\mathrm{e}}$, \\ Scott G. Palmer ${ }^{\mathrm{a}, \mathrm{e}}$, Sergei G. Sokolovski ${ }^{\mathrm{a}}$, Neil A. Stewart ${ }^{\mathrm{a}, \mathrm{e}}$, Edik U. Rafailov ${ }^{\mathrm{a}}$ \\ ${ }^{a}$ Photonics and Nanoscience Group, Division of Physics, School of Engineering, Physics and \\ Mathematics, University of Dundee, Dundee DD1 4HN, UK; ${ }^{b}$ State University - Education-Science- \\ Production Complex, Scientific-Educational Center of Biomedical Engineering, Oryol 302020, \\ Russia; "SPE "LAZMA" Ltd., Moscow 125252, Russia; ${ }^{\mathrm{d}}$ Priorov Central Research Institute of \\ Traumatology and Orthopaedics, Moscow 127299, Russia; 'Department of Imaging and Technology, \\ Ninewells Hospital, University of Dundee, Dundee DD1 9SY, UK
}

\begin{abstract}
Multi-functional laser non-invasive diagnostic systems, such as "LAKK-M", allow the study of a number of microcirculatory parameters, including blood microcirculatory index $\left(I_{m}\right)$ (by laser Doppler flowmetry, LDF) and oxygen saturation $\left(S_{t} O_{2}\right)$ of skin tissue (by tissue reflectance oximetry, TRO). Such systems may provide significant information relevant to physiology and clinical medicine. The aim of this research was to use such a system to study the synchronization of microvascular blood flow and oxygen saturation rhythms under normal and adaptive change conditions. Studies were conducted with 8 healthy volunteers -3 females and 5 males of 21-49 years. Each volunteer was subjected to basic 3 minute tests. The volunteers were observed for between 1-4 months each, totalling 422 basic tests. Measurements were performed on the palmar surface of the right middle finger and the forearm medial surface. Wavelet analysis was used to study rhythmic oscillations in LDF- and TRO-data. Tissue oxygen consumption (from arterial and venal blood oxygen saturation and nutritive flux volume) was calculated for all volunteers during "adaptive changes" as $(617 \pm 123 \mathrm{AU})$ and $(102 \pm 38 \mathrm{AU})$ with and without arteriovenous anastomoses (AVAs) respectively. This demonstrates increased consumption compared to normal (495 $\pm 170 \mathrm{AU})$ and $(69 \pm 40 \mathrm{AU})$ with and without AVAs respectively. Data analysis demonstrated the emergence of resonance and synchronization of rhythms of microvascular blood flow and oxygen saturation as an adaptive change in myogenic oscillation (vasomotion) resulting from exercise and potentially from psychoemotional stress. Synchronization of myogenic rhythms during adaptive changes suggest increased oxygen consumption resulting from increased microvascular blood flow velocity.
\end{abstract}

Keywords: laser Doppler flowmetry, tissue reflectance oximetry, vasomotion, oxygen consumption, adaptive changes

\section{INTRODUCTION}

The evaluation of stress-induced adaptive changes in the respiratory and circulatory systems of individuals could provide relevant information for studies in physiology and clinical medicine. In recent years, with the advent of multi-functional laser non-invasive diagnostic systems, such as the "LAKK-M" system (SPE "LAZMA" Ltd, Russia) ${ }^{1}$, it has become possible to conduct studies on a number of tissue parameters, including microvascular blood flow (by laser Doppler flowmetry, LDF) and oxygen saturation of skin tissue (by tissue reflectance oximetry, TRO) ${ }^{2}$.

The results of LDF measurements, representing "index of blood microcirculation $\left(I_{m}\right)$ " or "perfusion", assessed in conventional perfusion units (PU), reveal a complex, non-periodic process. This variable component contains information on the modulation of blood flow. Use of spectral signal processing algorithms on LDF-graphs for decoding and analysis provides information about the condition of vascular tone in terms of its contribution to the different mechanisms of micro-hemodynamic regulation ${ }^{3,4}$.

*a.v.dunaev@dundee.ac.uk; phone 441382 386571; dundee.ac.uk/elecengphysics/research/photonics/photonicsnanoscience 
Oscillatory processes play an important role in the function of the tissue microcirculation system. Several frequency ranges of blood flow oscillations in microvascular networks, each of a different regulatory origin, have been identified (endothelial, neurogenic, myogenic, etc.) ${ }^{5-7}$. Many medical publications are devoted to the study of myogenic oscillations, because they characterize the state of pre-capillary sphincters, which play an important role in the regulation of blood flow ${ }^{8-10}$.

The TRO method determines relative blood volume $\left(V_{b}\right.$, in percent) in microcirculation in the surface layers of the soft tissues (skin, mucous membranes of the organs) and tissue oxygen saturation $\left(S_{t} O_{2}\right.$, in percent) in the microvasculature in the inspected area of biological tissue. There are few spectral processing algorithms for these recorded signals $\left(S_{t} O_{2}-\right.$ and $V_{b}$-graphs) ${ }^{11,12}$, and there are limited publications studying the relationships between LDF- and $S_{t} O_{2}$-graphs ${ }^{13,14}$. In isolated cases this has been used to assess vasomotion and myogenic rhythms for perfusion and tissue oxygen saturation, for example ${ }^{15}$. We propose that analysis of oscillation signals recorded by TRO according to the frequency ranges, similar to LDF-graphs, is of practical interest in studying the microcirculation of blood, as the relationships between LDF and TRO attract increasing attention from researchers in this field.

The aim of this research was to use LDF- and TRO-graphs to investigate tissue respiration and the synchronization of microvascular blood flow and oxygen saturation rhythms under normal conditions and during adaptive changes.

\section{THE METHOD OF RESEARCH}

In this study we used a "LAKK-M" system, which, besides LDF and TRO, contains pulse oximetry and laser fluorescence diagnostic channels ${ }^{1}$. This system utilizes near-infrared $(1064 \mathrm{~nm})$, red $(640 \mathrm{~nm})$ and green $(532 \mathrm{~nm})$ lasers for LDF- and TRO-channels and performs simultaneous recording of the $I_{m}, S_{t} O_{2}$ and $V_{b}$ parameters in a tissue volume of approximately 3-5 $\mathrm{mm}^{3}$. Studies, of different durations, were conducted with 8 healthy volunteers (no history of cardiovascular disease) aged 21-49 years, comprising 3 females and 5 males. These studies were conducted by simultaneously recording parameters of $\operatorname{LDF}\left(I_{m}\right)$, TRO $\left(S_{t} O_{2}\right)$ and pulse oximetry $\left(S_{a} O_{2}\right.$ - arterial blood saturation with oxygen). In order to assess the $I_{m}$ and the $S_{t} O_{2}$ oscillatory component, spectral wavelet analysis of oscillations was used (software LDF 3.0.2.384, LAZMA, Russia). This program uses a continuous wavelet transform, with the Morle complex valued wavelet being used as the analyzing wavelet ${ }^{16}$. The study was performed at an ambient temperature of $21-22^{\circ} \mathrm{C}$ in a sitting position after a $30 \mathrm{~min}$ rest. The measurements were performed on skin pad (palmar surface) of right middle finger (Fig. 1a). This area was chosen because it is rich in arteriovenous anastomoses (AVAs) and variability of the LDF signal is less than in tissue with fewer shunts ${ }^{17}$. It should be emphasized that this area is regulated almost exclusively by the autonomic nervous system and is very sensitive to adaptive changes. In addition, studies were conducted in an area almost completely devoid of AVAs - the lower arm's medial surface (the skin without AVAs), characterized by greater nutritive blood flow (Fig. 1b).

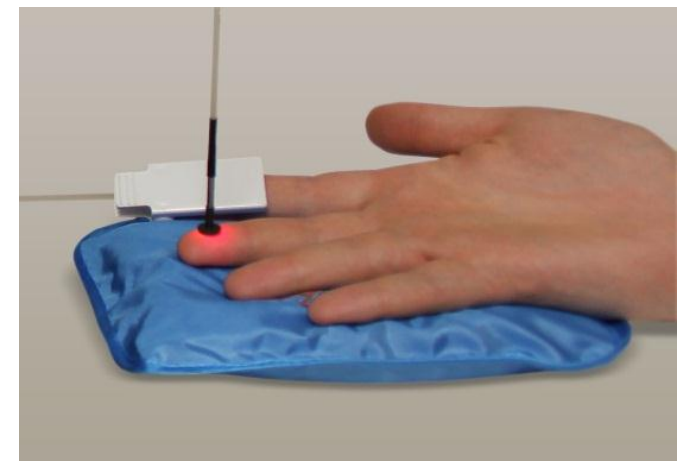

a)

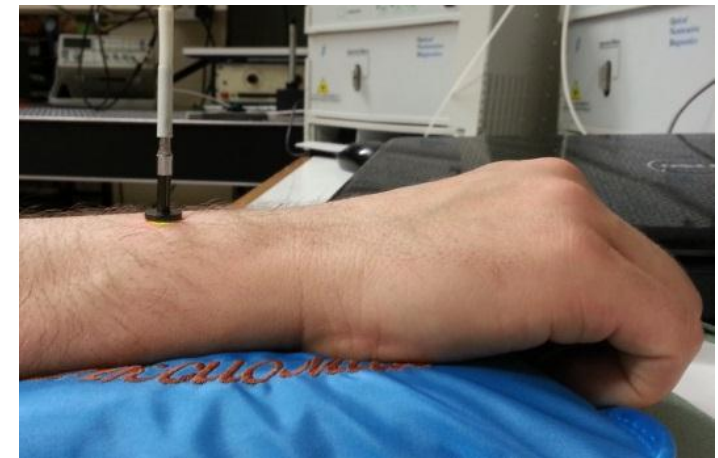

b)

Figure 1. Skin areas of study: with AVAs (a) and without AVAs (b).

The male participant of 36 years (volunteer №1) was studied over the course of 6 months, totaling 100 records in the skin with AVAs: 60 basic tests for 3 min, plus 20 "before and after" records to monitor the effects of exercise, in this case swimming $(500 \mathrm{~m})$. This volunteer's studies have also been conducted in the skin without AVAs. The female 
participant (volunteer №2) was studied over the course of 1 month, totaling 40 studies only in the skin with AVAs: 20 basic tests for 3 minutes plus 20 tests with occlusion for 1 min followed by a 3 min post occlusion period. For the remaining volunteers, only basic tests were performed on both points of interest (skin with and without AVAs) and they were not subjected to additional stress. Wavelet analysis was performed on 5 rhythmic components (oscillations) of $I_{m^{-}}$ and $S_{t} O_{2}$-records, namely: endothelial $(0.0095-0.02 \mathrm{~Hz})$; neurogenic $(0.02-0.06 \mathrm{~Hz})$; myogenic $(0.06-0.16 \mathrm{~Hz})$; breathing $(0.16-0.4 \mathrm{~Hz})$ and pulse $(0.4-1.6 \mathrm{~Hz})^{5,18,19}$. The typical form of perfusion and tissue oxygen saturation are shown in Fig $2 \mathrm{a}$ and the results of the wavelet analysis for them during the basic test are presented in Fig. $2 \mathrm{~b}$.
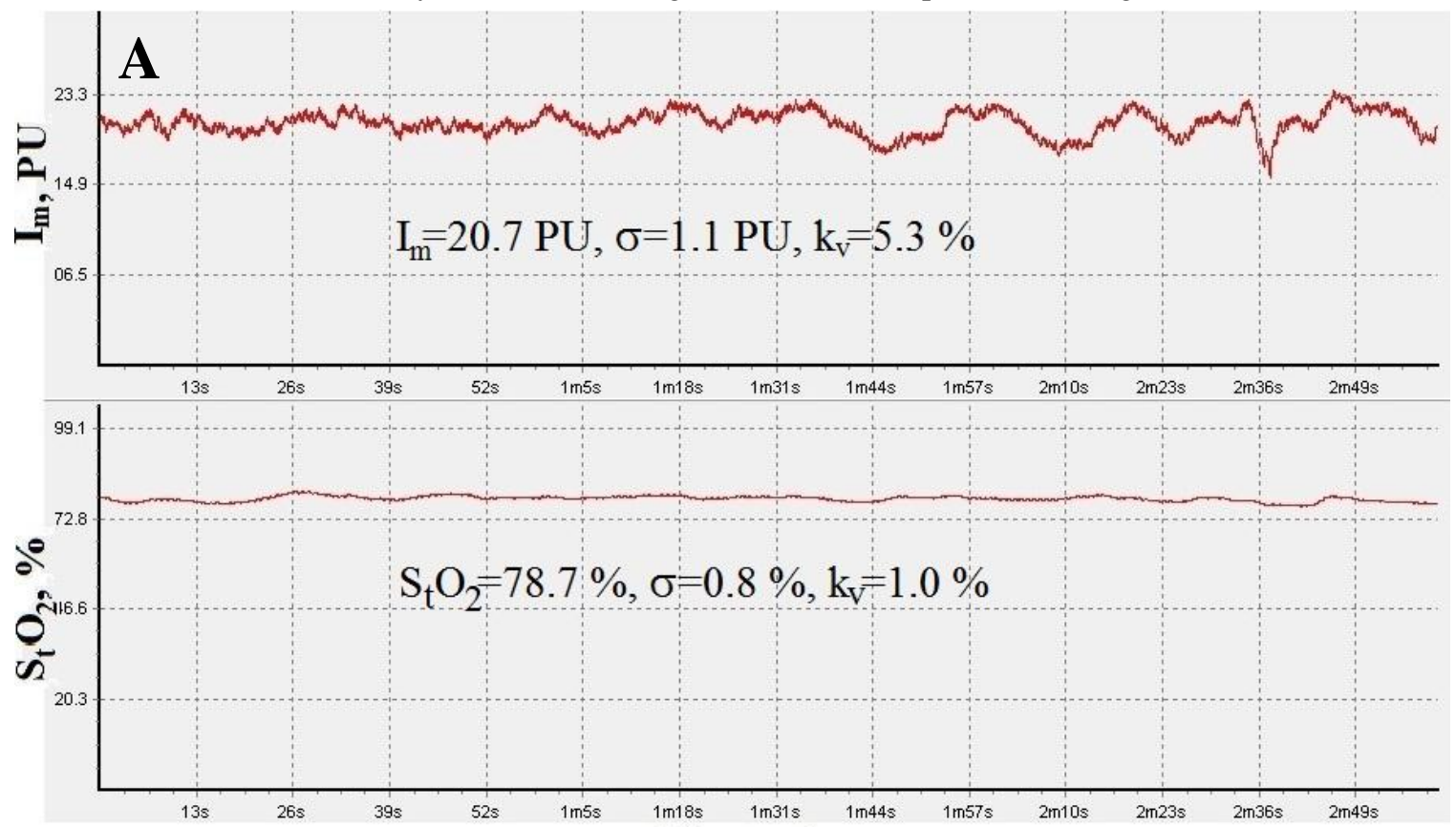

Time, $\min$
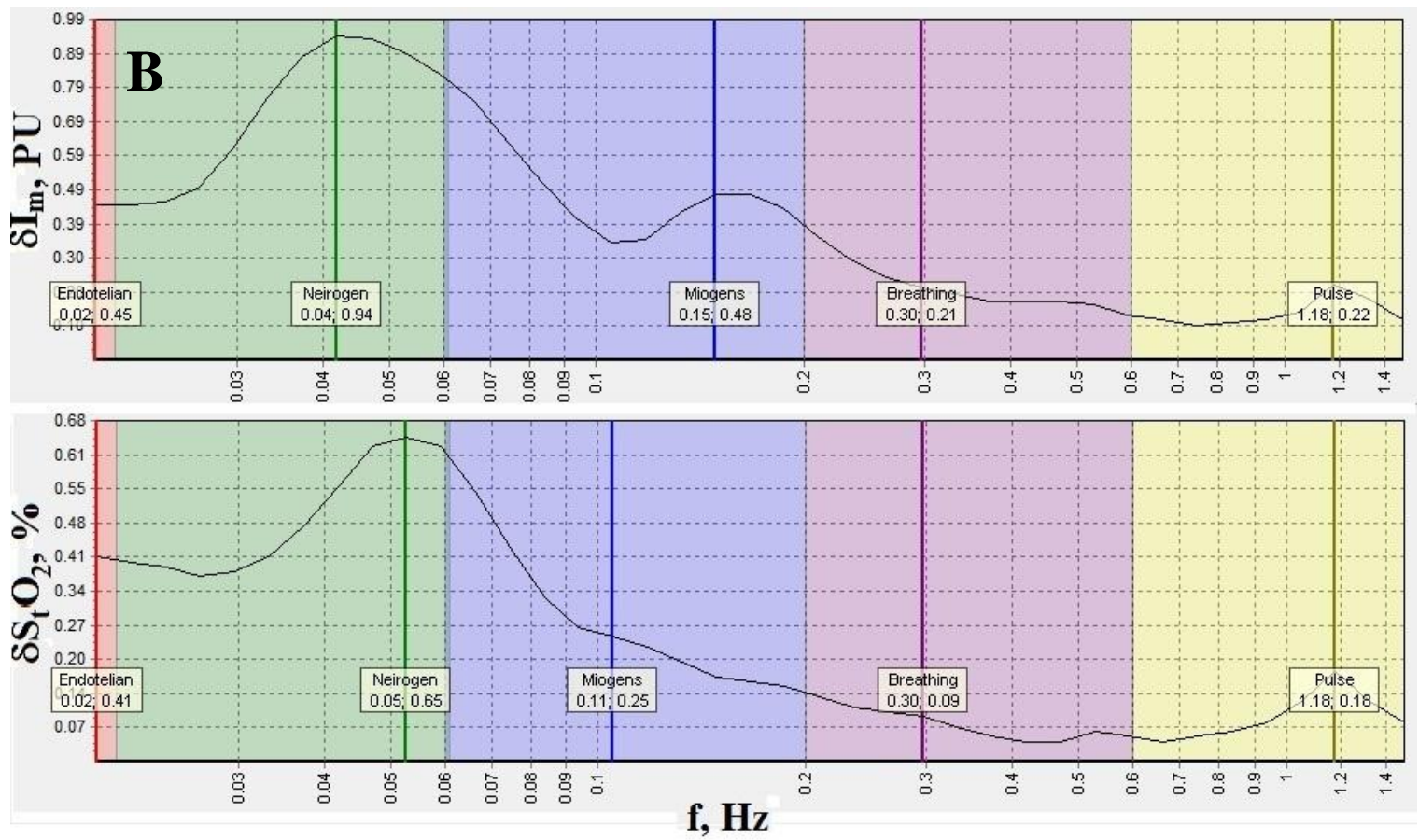

Figure 2. The typical form of perfusion and tissue oxygen saturation graphs of the skin area with AVAs for volunteer №1 (a), where $\sigma$ - standard deviation, $k_{v}$ - coefficient of variation, and wavelet analysis results following such basic tests (b), where $\delta I_{m}$ - amplitude of perfusion oscillations, $\delta S_{t} O_{2}$ - amplitude of oxygen saturation oscillations. Furthermore, in (b), a line is used to represent the amplitude oscillation of microvascular blood flow ( $\left.\delta I_{m}=0.48 \mathrm{PU}\right)$ at a frequency of $f_{m}=0.149 \mathrm{~Hz}$ and tissue oxygen saturation $\left(\delta S_{t} O_{2}=0.25 \%\right)$ at a frequency of $f_{m}=0.105 \mathrm{~Hz}$ for myogenic rhythms. 
During every test, the psychoemotional state of the volunteer was recorded as either normal or under emotional stress. In the case of volunteer №2, physiological stress was induced through occlusion tests, while for volunteer №1 this was achieved by exercise (swimming), which can be considered as stress simulation.

Of particular interest is the analysis and comparison of oxygen consumption in tissue under normal conditions and during adaptive changes, associated with a sympathetic vasomotor reflex (synchronization and resonance of myogenic oscillation in perfusion and tissue oxygen saturation). This is especially noteworthy as a relationship between the activation of vasomotion and oxygen consumption has been previously reported ${ }^{20}$. Following the methodology explained in the article ${ }^{21}$ and from spectral wavelet analysis of $I_{m}$ - and $S_{t} O_{2}$-graphs, we calculated the extraction and consumption of oxygen in tissue for all 8 volunteers.

Oxygen extraction $(O E)$, assessed in arbitrary units (AU), was calculated as follows:

$$
O E=\left(S_{a} O_{2}-S_{v} O_{2}\right) / S_{a} O_{2}
$$

where $\mathrm{S}_{v} \mathrm{O}_{2}$ - venous blood oxygen saturation, calculated using spectral wavelet analysis of $\mathrm{S}_{t} \mathrm{O}_{2}$ oscillations. We also analyzed the amplitude of oscillations of cardiac $\left(\delta S_{t} O_{2}\right)_{c}$ and respiratory rhythms $\left(\delta S_{t} O_{2}\right)_{r}$. If the $\left(\delta S_{t} O_{2}\right)_{c} /\left(\delta S_{t} O_{2}\right)_{r}$ ratio $\leq$ 1 , then $\mathrm{S}_{v} \mathrm{O}_{2}$ is taken to be equal to $\mathrm{S}_{t} \mathrm{O}_{2}$. This variant predominates in most cases of recordings from the skin without AVAs ${ }^{22}$. If the $\left(\delta S_{t} O_{2}\right)_{c} /\left(\delta S_{t} O_{2}\right)_{r}$ ratio $>1$, then:

$$
S_{v} O_{2}=S_{t} O_{2} /\left(\left(\delta S_{t} O_{2}\right)_{c} /\left(\delta S_{t} O_{2}\right)_{r}\right.
$$

This variant predominates in most cases of recordings from the skin with AVAs. In the cases of resonance oscillations in the active frequency bands (for example, in the myogenic range during adaptive changes), the cardiac and/or respiratory rhythm amplitudes may not be expressed in the spectrum, and the $\mathrm{S}_{v} \mathrm{O}_{2}$ calculation has some specific features. In the cases of resonance of oscillations in the total myogenic and respiratory bands, $S_{\nu} O_{2}=S_{t} O_{2}$. In the skin zones with AVAs, an additional confirmation using the bypass index $(B I)$ for $S_{t} O_{2}$ is necessary ${ }^{21}$ :

$$
\mathrm{S}_{v} \mathrm{O}_{2}=\mathrm{S}_{t} \mathrm{O}_{2} / \mathrm{BI}\left(\mathrm{S}_{t} \mathrm{O}_{2}\right)
$$

where:

$$
B I\left(S_{t} O_{2}\right)=1+\left(\delta S_{t} O_{2}\right)_{n} /\left(\delta S_{t} O_{2}\right)_{m}
$$

where $\left(\delta S_{t} O_{2}\right)_{n}$ and $\left(\delta S_{t} O_{2}\right)_{m}$ - amplitudes of oscillations of neurogenic and myogenic rhythms respectively.

Oxygen consumption $(O C)$, assessed in arbitrary units (AU), was calculated as follows:

$$
O C=I_{m n} \cdot\left(S_{a} O_{2}-S_{v} O_{2}\right)
$$

where $I_{m n}$ - the nutritive blood flow value was calculated according to the equation:

$$
I_{m n}=I_{m} / B I\left(I_{m}\right)
$$

where $B I\left(I_{m}\right)$ - bypass index calculated for skin with AVAs similarly to formula (4), but only using perfusion data. For skin zones without AVAs:

$$
B I\left(I_{m}\right)=\left(\delta I_{m}\right)_{\max } /\left(\delta I_{m}\right)_{m}
$$

where $\left(\delta I_{m}\right)_{\max }$ is the maximum amplitude of the dominant oscillations in the active range of frequencies up to $0.15 \mathrm{~Hz}$ and $\left(\delta I_{m}\right)_{m}$ - amplitude of oscillations of myogenic rhythms.

Accordingly, the calculation of oxygen consumption in cases with and without adaptive changes (for synchronization and resonance of myogenic oscillations in perfusion and oxygen saturation) were processed separately. It should be 
noted that the $O C$ equation includes the perfusion rate value, thus the $O C$ value (calculated according to Fick's principle) reflects the oxygen consumption rate.

The following parameter has also been calculated and analyzed:

$$
\Delta f_{m}=f_{m}\left(S_{t} O_{2}\right)-f_{m}\left(I_{m}\right),
$$

where $f_{m}$ - myogenic oscillation frequency of perfusion and tissue oxygen saturation, respectively.

Data presented in the text are means \pm SD. Statistical analysis was performed by OriginPro 8 SRO version v.8.0724 with data sets tested for normality by the Kolmogorov-Smirnov and Shapiro-Wilk tests. For normally distributed data, group comparisons were made by carrying out a parametric unpaired $t$-test. For the obtained non-normal data distribution of data, Mann-Whitney test nonparametric statistics was used to compare the two groups (normal and with conditions of adaptive changes).

\section{RESULTS AND DISCUSSION}

Analysis of the data shows the emergence of synchronized rhythms in microvascular blood flow and oxygen saturation within the myogenic oscillation range (vasomotion) during adaptive changes - for example, stressful situations or response to physical exercise. The typical perfusion and tissue oxygen saturation data, along with the results of wavelet analysis of these parameters during adaptive changes for volunteer 1 are presented in Fig. 3.

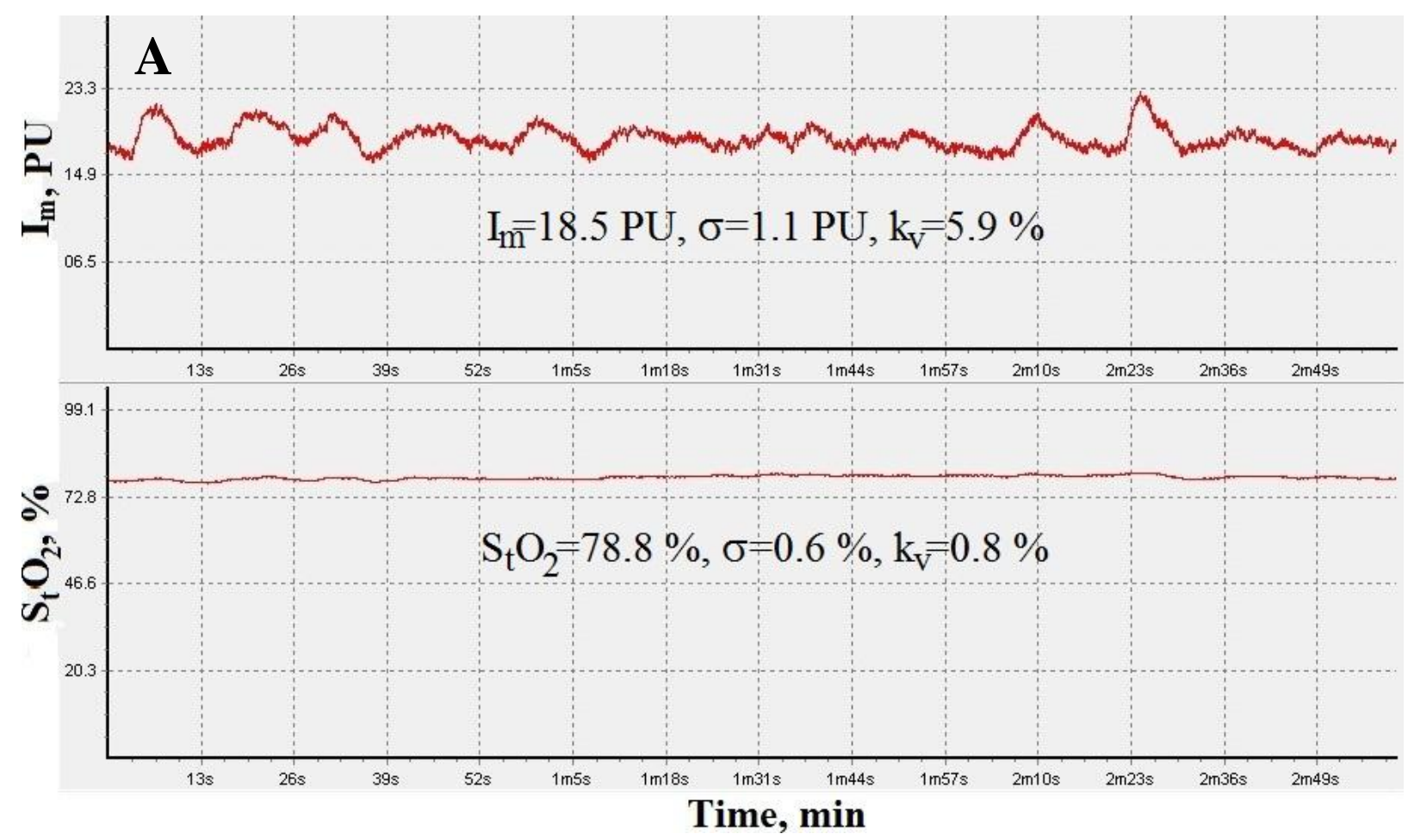



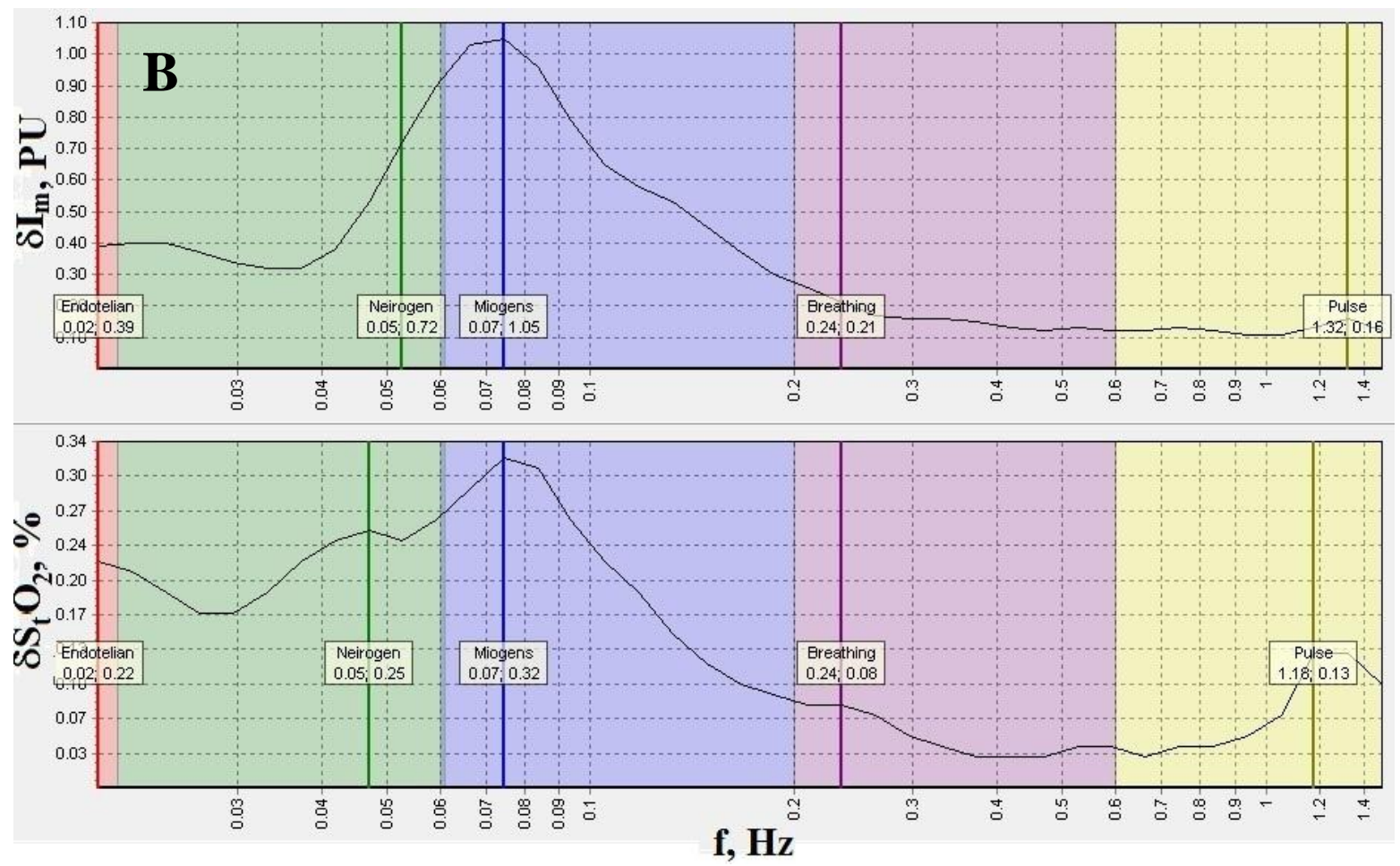

Figure 3. Perfusion and oxygen saturation graphs in cases of myogenic oscillation of the skin area with AVAs for volunteer №1 (A) and typical example of resonance and synchronized rhythms $\left(f_{m}=0.074 \mathrm{~Hz}\right)$ of microvascular blood flow $\left(\delta I_{m}=1.05\right.$ $\mathrm{PU}$ ) and oxygen saturation $\left(\delta S_{t} \mathrm{O}_{2}=0.32 \%\right)$ within the range of only myogenic oscillation (vasomotion) during adaptive changes (B).

The parameters for calculating oxygen extraction and consumption for all volunteers were obtained using the approaches detailed in the methods section above. The results of measurements and calculations for both areas studied (skin with and without AVAs) for all the 8 volunteers are shown in Table 1.

Table 1. The results of measurements and calculations for all 8 volunteers.

\begin{tabular}{|c|l|c|c|c|c|}
\hline \multirow{2}{*}{ № } & \multicolumn{1}{|c|}{ Parameters } & \multicolumn{2}{|c|}{ Skin with AVAs } & \multicolumn{2}{c|}{ Skin without AVAs } \\
\cline { 3 - 6 } & & $\begin{array}{c}\text { Norm } \\
(\mathbf{n = 1 8 7})\end{array}$ & $\begin{array}{c}\text { With adaptive } \\
\text { changes }(\mathbf{n = 6 0})\end{array}$ & $\begin{array}{c}\text { Norm } \\
(\mathbf{n = 1 2 8})\end{array}$ & $\begin{array}{c}\text { With adaptive } \\
\text { changes (n=26) }\end{array}$ \\
\hline 1 & $I_{m}$ (total), PU & $21.0 \pm 3.1$ & $21.4 \pm 3.4$ & $2.5 \pm 0.8$ & $2.8 \pm 0.9$ \\
\hline 2 & $I_{m n}($ nutritive), PU & $8.6 \pm 0.5$ & $11.1 \pm 2.2^{*}$ & $1.7 \pm 0.8$ & $2.8 \pm 0.8^{* *}$ \\
\hline 3 & $S_{a} O_{2}, \%$ & $98.1 \pm 0.4$ & $97.9 \pm 0.4$ & $97.9 \pm 0.4$ & $97.8 \pm 0.6$ \\
\hline 4 & $S_{t} O_{2}, \%$ & $78.3 \pm 4.7$ & $77.7 \pm 5.7$ & $66.2 \pm 9.3$ & $61.9 \pm 7.3$ \\
\hline 5 & $S_{\nu} O_{2}, \%$ & $41.6 \pm 13.7$ & $41.9 \pm 6.1$ & $58.2 \pm 12.7$ & $61.3 \pm 7.3$ \\
\hline 6 & $V_{b}, \%$ & $10.2 \pm 1.8$ & $9.9 \pm 1.5$ & $6.3 \pm 1.7$ & $6.0 \pm 1.4$ \\
\hline 7 & $B I\left(I_{m}\right), \mathrm{AU}$ & $2.5 \pm 0.5$ & $1.9 \pm 0.2^{* *}$ & $1.6 \pm 0.6$ & $1.0 \pm 0.06^{* *}$ \\
\hline 8 & $B I\left(S_{t} O_{2}\right), \mathrm{AU}$ & $2.7 \pm 0.7$ & $1.9 \pm 0.2^{* *}$ & $2.4 \pm 1.6$ & $1.0 \pm 0.2^{* *}$ \\
\hline 9 & $O E, \mathrm{AU}$ & $0.58 \pm 0.14$ & $0.57 \pm 0.06$ & $0.41 \pm 0.13$ & $0.37 \pm 0.07$ \\
\hline 10 & $O C, \mathrm{AU}$ & $495 \pm 170$ & $617 \pm 123^{* *}$ & $69 \pm 40$ & $102 \pm 38^{* *}$ \\
\hline
\end{tabular}

Note: There is significant difference $(p>0.05)$ observed from normal state, calculated by a $t$-test $(*)$ and by the MannWhitney test $(* *)$.

The human skin contains functionally distinct zones, differing in morphological properties and the regulation of microvascular blood flow. These can be classified as with and without the presence of AVAs. The zones with AVAs are 
functionally tied to implementation of the thermoregulatory homeostasis and are almost exclusively regulated by the sympathetic adrenergic nervous system. Additionally, the values of perfusion and intravascular pressure of the skin microvessels is generally higher in regions containing AVAs. The zones of skin without AVAs are characterized by lower blood flow in microvessels and a higher contribution of the venous component. During adaptive changes, a significant increase in the nutritive perfusion $\left(I_{m n}\right)$ is observed in the zones with AVAs (from 8.6 \pm 0.5 PU to 11.1 $\pm 2.2 \mathrm{PU}$, $p>0.05$ ). Oxygen extraction did not change in the zones with and without AVAs. Increasing oxygen consumption was therefore due to an increase in perfusion rather than an increase in OE, thus adaptive changes naturally lead to the intensification of oxygen consumption in zones with AVAs (from 495 \pm 170 AU to $617 \pm 123 \mathrm{AU}, p>0.05$ ). More cases of synchronization of myogenic rhythms in microvascular blood flow and oxygen saturation were registered in zones with AVAs, this is most likely because of the large numbers of autonomic nerves, which are very sensitive to adaptive changes.

The results from our studies on adaptive changes (stress- or exercise-induced) support our hypothesis that during resonance and synchronization of blood flow and oxygen saturation rhythms via myogenic oscillation there is increased tissue oxygen consumption compared with normal conditions. Thus the level of extraction of oxygen from blood remains unchanged. Therefore, we suggest that the bypass index may be used as a marker of adaptive changes (during stress conditions), calculated based on perfusion and tissue oxygen saturation.

The increase in amplitude of myogenic rhythm reflects a modulation of the hydrostatic pressure in the capillaries, resulting in an increase in diffusion of oxygen into the tissues, hence the changes in tissue oxygen saturation. Time shifts and frequency characteristics are obviously specific to particular individuals. For example, volunteer №1 registered 41 cases of synchronization (21 cases under emotional stress and 20 cases induced by exercise), volunteer №2 only registered 5 cases of synchronization of vasomotion (including cases obtained as a result of occlusion tests) and volunteer №3 registered 4 cases of adaptive changes. Analysis of recorded time fragments under normal conditions, during stress and following exercise presented distinct differences in the frequency of myogenic oscillations in perfusion and tissue oxygen saturation $\left(\Delta f_{m}\right)$. In the normal state for all volunteers, this difference is almost always negative, because myogenic oscillations in perfusion typically display greater frequency than those for oxygen saturation. For example, the mean value for volunteer №1: $0.006 \mathrm{~Hz}$; for volunteer №2: $0.022 \mathrm{~Hz}$; for volunteer №3: $0.019 \mathrm{~Hz}$. But during adaptive changes (in stressful situations - physical or emotional stress) when there is synchronization of myogenic oscillations, oxygen saturation is more intensive, giving $\Delta f_{m}$ results which are generally positive. Occasionally, complete synchronization will be achieved when the frequency of both myogenic oscillations coincide. For example, this case is presented in Fig.4.

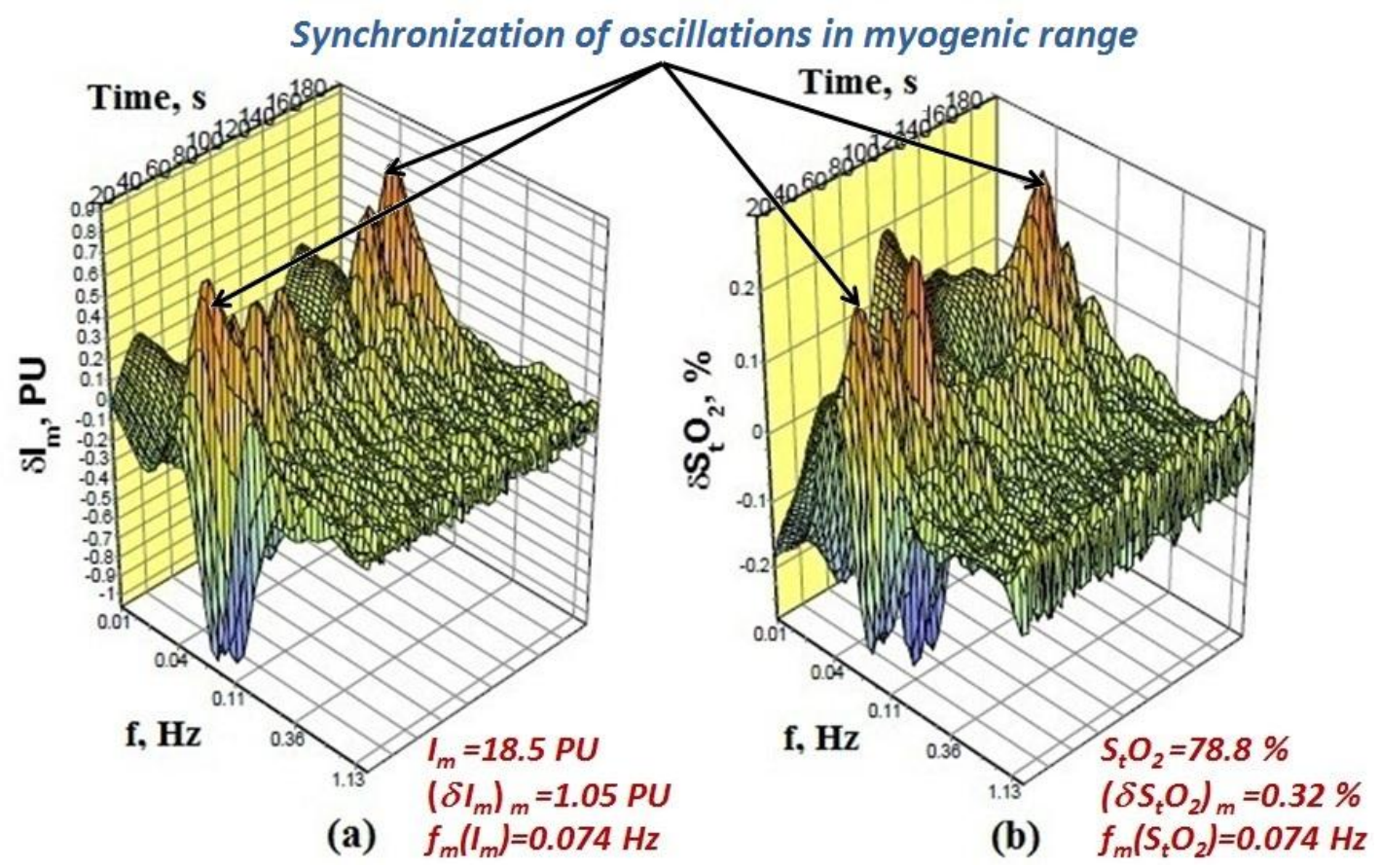

Figure 4. Typical example of the 3D wavelet analysis of resonating and synchronized myogenic rhythms of microvascular blood flow (a) and oxygen saturation (b) during adaptive changes. 
The values of $\Delta f_{m}$ during adaptive changes registered for the volunteers 1-3 were $0.027 \mathrm{~Hz}, 0.015 \mathrm{~Hz}$ and $0.040 \mathrm{~Hz}$, respectively. It is worth noting that in studies of the effect of exercise (swimming) on blood flow and tissue oxygen saturation (especially at the initial stage of training) full synchronization of myogenic oscillations was achieved $\left(\Delta f_{m}=0\right)$.

Data analysis has demonstrated the emergence of resonance and synchronized rhythms of microvascular blood flow and oxygen saturation as an adaptive change in myogenic oscillation (vasomotion) resulting from exercise and potentially from psychoemotional stress. Perhaps one explanation for the origin of this phenomenon is that the synchronization of myogenic rhythms facilitates maximum oxygen delivery to tissue following physical or emotional stress.

\section{CONCLUSION}

The data obtained show behavioral differences in myogenic oscillations in the ordinary state of the body (normality) and during episodes of sympathoadrenal activation (e.g. emotional stress). Normally, all systems of the body (including blood circulation, respiration, metabolism, etc.) work in different phases and frequencies, exhibiting non-linearity and independence. Synchronization of myogenic rhythms during adaptive changes may lead to increased oxygen consumption resulting from increased microvascular blood flow velocity. The data above suggests that adaptive changes naturally lead to the intensification of oxygen consumption in zones with AVAs due to an increased perfusion. Furthermore, as these zones have rich autonomic innervation, they are very sensitive to adaptive change leading to them having the highest incidence of myogenic rhythm synchronization of microvascular blood flow and oxygen saturation. During adaptive changes (under particular emotional stress, etc.), synchronization increases, reducing the freedom of microvascular blood flow regulation.

Ultimately, our suggested approaches for the use of the laser technology in investigating tissue respiration and skin microhaemocirculation under adaptive changes, through the monitoring of synchronization of blood flow and oxygen saturation rhythms observed within the data, have shown themselves to be highly informative and present interesting prospects for further investigation as a potential diagnostic methodology relevant to vascular function.

\section{ACKNOWLEDGMENTS}

This work was supported by the European Community's Seventh Framework Programme (FP7-People-2009-IAPP) under Grant Agreement no. 251531 MEDILASE.

\section{REFERENCES}

[1] Rogatkin, D. A., Sokolovski, S. G., Fedorova, K. A., Sidorov, V. V., Stewart, N. Z. and Rafailov, E. U., "Basic principles of design and functioning of multifunctional laser diagnostic system for non-invasive medical spectrophotometry," Proc. SPIE. 7890, 78901H1 (2011).

[2] Dunaev, A. V., Sidorov, V. V., Stewart, N. A., Sokolovski, S. G. and Rafailov, E. U., "Laser reflectance oximetry and Doppler flowmetry in assessment of complex physiological parameters of cutaneous blood microcirculation," Proc. SPIE. 8572, 857205 (2013).

[3] Bracic, M. and Stefanovska, A., "Wavelet-based analysis of human blood-flow dynamics," Bulletin of Mathematical Biology, 60(5), 919-935 (1998).

[4] Krupatkin, A. I. and Sidorov, V. V., [Laser Doppler flowmetry of blood microcirculation], Meditcina-Press, Moscow (2005).

[5] Kvandal, P., Landsverk, S. A., Bernjak, A., Stefanovska, A., Kvernmo, H. D. and Kirkeboen, K. A., "Lowfrequency oscillations of the laser Doppler perfusion signal in human skin," Microvascular Research, 72(3), 120127 (2006).

[6] Krupatkin, A. I., "The Problem of Information Value in Microvascular Networks," Human Physiology, 37(3), 312-317 (2011). 
[7] Kvernmo, H. D., Stefanovska, A., Kirkeboen, K. A. and Kvernebo, K., "Oscillations in the human cutaneous blood perfusion signal modified by endothelium-dependent and endothelium-independent vasodilators," Microvascular Research, 57(3), 298-309 (1999).

[8] Schmidt-Lucke, C., Borgstrom, P. and Schmidt-Lucke, J. A., "Low frequency flowmotion/(vasomotion) during patho-physiological conditions," Life Sciences, 71(23), 2713-2728 (2002).

[9] Rossi, M., Carpi, A., Galetta, F., Franzoni, F. and Santoro, G., "Skin vasomotion investigation: A useful tool for clinical evaluation of microvascular endothelial function?," Biomedicine \& Pharmacotherapy, 62(8), 541-545 (2008).

[10] Schmiedel, O., Schroeter, M. L. and Harvey, J. N., "Microalbuminuria in Type 2 diabetes indicates impaired microvascular vasomotion and perfusion," American Journal of Physiology - Heart and Circulatory Physiology, 293(6), H3424-H3431 (2007).

[11] Stefanovska, A., "Dynamics of blood oxygenation gives better insight into tissue hypoxia than averaged values," American Journal of Physiology - Heart and Circulatory Physiology, 296(5), H1224-H1226 (2009).

[12] Thorn, C. E., Matcher, S. J., Meglinski, I. V. and Shore, A. C., "Is mean blood saturation a useful marker of tissue oxygenation?," American Journal of Physiology - Heart and Circulatory Physiology, 296(5), H1289-H1295 (2009).

[13] Bernjak, A., Stefanovska, A., McClintock, P. V. E., Owen-Lynch, P. J. and Clarkson, P. B. M., "Coherence between fluctuations in blood flow and oxygen saturation," Fluctuation and Noise Letters, 11(1), (2012).

[14] Tyrrell, J., Thorn, C., Shore, A., Campbell, S. and Curnow, A., "Oxygen saturation and perfusion changes during dermatological methylaminolaevulinate photodynamic therapy," British Journal of Dermatology, 165(6), 13231331 (2011).

[15] Thorn, C. E., Kyte, H., Slaff, D. W. and Shore, A. C., "An association between vasomotion and oxygen extraction," American Journal of Physiology-Heart and Circulatory Physiology, 301(2), H442-H449 (2011).

[16] Tankanag, A. V. and Chemeris, N. K., "A method of adaptive wavelet filtering of the peripheral blood flow oscillations under stationary and non-stationary conditions," Physics in Medicine and Biology, 54(19), 5935-5948 (2009).

[17] Salerud, E. G., Tenland, T., Nilsson, G. E. and Oberg, P. A., "Rhythmical variations in human-skin blood-flow," International Journal of Microcirculation-Clinical and Experimental, 2(2), 91-102 (1983).

[18] Stefanovska, A., Bracic, M. and Kvernmo, H. D., "Wavelet analysis of oscillations in the peripheral blood circulation measured by laser Doppler technique," IEEE Transactions on Biomedical Engineering, 46(10), 12301239 (1999).

[19] Krupatkin, A. I., "Blood flow oscillations at a frequency of about $0.1 \mathrm{~Hz}$ in skin microvessels do not reflect the sympathetic regulation of their tone," Human Physiology, 35(2), 183-191 (2009).

[20] Kislukhin, V. V., "Regulation of oxygen consumption by vasomotion," Mathematical Biosciences, 191(1), 101108 (2004).

[21] Krupatkin, A. I., "Noninvasive estimation of human tissue respiration with wavelet-analysis of oxygen saturation and blood flow oscillations in skin microvessels," Human Physiology, 38(4), 396-401 (2012).

[22] Krupatkin, A. I., "Cardiac and respiratory oscillations of the blood flow in microvessels of the human skin," Human Physiology, 34(3), 323-329 (2008). 